\title{
5 Forschungfrage
}

Die aus der Forschungslücke abzuleitende Frage stellt sich so dar:

„Lässt sich durch eine Integration von ACT-R, ,Integrated Theory of Mind“', dem Vierebenenmodell der Persönlichkeiten und dem Modell zur hierarchischen Kontrolle unter Abstützung auf den REST-Zustand sowie das CREATES-Modell der Kreativität eine Erklärung auf die Frage finden, wie aus dem Denken Neues und somit Kreativität entstehen kann?““

Um dies Forschungsfrage zu beantworten, wird es weiters nötig sein, sich folgende Fragen zu stellen:

- Welche gemeinsamen Elemente verbinden ACT-R, Integrated Theory of Mind, das Vierebenenmodell der Persönlichkeiten und das Modell zur hierarchischen Kontrolle?

- Wie kann man die Integration von ACT-R, Integrated Theory of Mind, dem Vierebenenmodell der Persönlichkeiten und dem Modell zur hierarchischen Kontrolle durchführen? 